\title{
Antioxidant, biofilm inhibition and mutagenic activities of newly substituted fibrates
}

\author{
Asma Sheikh ${ }^{1 \star}$, Zia-Ur-Rehman ${ }^{2}$, Muhammad Imran ${ }^{1}$, Zaid Mahmood ${ }^{1}$ \\ ${ }^{1}$ Institute of Chemistry, University of the Punjab, Lahore, ${ }^{2}$ Applied Chemistry Research Centre, PCSIR, Labs, Complex, Lahore, \\ Pakistan
}

${ }^{\star}$ For correspondence: Email: asmasheikhso@yahoo.com

\begin{abstract}
Purpose: A series of benzylidene-2-(4-bromophenoxy)-2-methyl propane hydrazides (1-10) were synthesized and assay them for their biofilm inhibition, antioxidant and mutagenic. .

Methods: All derivatives were prepared by condensation of various substituted benzaldehyde and acetophenones with 2-(4-bromorophenoxy)-2-methyl propane hydrazide, which was itself prepared by hydrazinolysis of ethyl-2-(4-bromophenoxy)-2-methyl propanoate and were characterized by FTIR, ${ }^{1} \mathrm{H}$ $N M R{ }^{13} \mathrm{C} N M R$, mass spectrometry. They were screened for their in-vitro anti-oxidant, biofilm inhibition and mutagenicity by established methods.

Results: Anti-oxidant results revealed that the electron donating group enhanced the scavenging ability of the compounds as seen in compounds $\mathbf{4 b}$, $\mathbf{4} \boldsymbol{h}$ and $\mathbf{4 i}$. In biofilm inhibition studies, all compounds were more active against Gram -ive bacterial strain when compared to gram +ive strain. The mutagenicity assay results indicate that the compound having chloro group substitution is mutagenic.

Conclusion: The benzylidine compounds of 2-(4-bromophenoxy)-2-methyl hydrazide possessing electron donating substituents exhibit superior activities to the electron withdrawing group substituents.
\end{abstract}

Keywords: Fibrate derivatives, Anti-oxidant, Biofilm inhibition, Mutagenic activity

\begin{abstract}
This is an Open Access article that uses a fund-ing model which does not charge readers or their institutions for access and distributed under the terms of the Creative Commons Attribution License (http://creativecommons.org/licenses/by/4.0) and the Budapest Open Access Initiative (http://www.budapestopenaccessinitiative.org/read), which permit unrestricted use, distribution, and reproduction in any medium, provided the original work is properly credited.
\end{abstract}

Tropical Journal of Pharmaceutical Research is indexed by Science Citation Index (SciSearch), Scopus, International Pharmaceutical Abstract, Chemical Abstracts, Embase, Index Copernicus, EBSCO, African Index Medicus, JournalSeek, Journal Citation Reports/Science Edition, Directory of Open Access Journals (DOAJ), African Journal Online, Bioline International, Open-J-Gate and Pharmacy Abstracts

\section{INTRODUCTION}

Fibrates are a class of drugs having a stable phenoxy isobutyric acid (pharmacophore moiety) [1] used for the treatment of coronary heart disease, ischemic cerebrovascular diseases and peripheral vascular disorder [2]. Furthermore, these fibrates inhibit the accumulation of lipids in arteries and stimulate the production of high density lipoprotein cholesterol [3]. Guido Bargellini introduced the synthesis of fibric acid compound [4] and found it very responding to human bio-chemical system for the treatment of dyslipidemia. Later, many other fibrates like Bezafibrate, Clofibrate, Fenofibrate and Ciprofibrate [5] were reported. In the last decade, scientists have coupled this moiety with various heterocyclic nuclei such as benzoxazole [6], indole-fibrate [7], amide-fibrate [8], tetrazole [9] and oxadizole [10] to get more potent biologically active compound.

Keeping in view the stable structure of fibrates, their tremendous biological activities and 
absence of disproportionation, the ethyl 2-(4bromophenoxy)-2-methyl propanoate having fibrate moiety was synthesized and converted into biologically active azomethine compounds and investigated their antioxidant, biofilm inhibition and mutagenic properties with respect to the presence substituents and azomethine linkage.

\section{EXPERIMENTAL}

\section{Chemicals, reagents and equipment}

All the chemicals were purchased from E. Merck, Sigma Aldrich and Fluka and were used without purification. Bruker Tensor 27 spectrophotometer used for IR spectra (in $\mathrm{KBr}$ disk). For ${ }^{1} \mathrm{H}$ NMR Bruker Avance-III instrument was used. TMS (trimethyl silane) was used as an internal standard and chemical shifts were reported in ppm. For elemental analysis Perkin Elmer 2400-CHNS Analyzer was used, while Melting points were record on a Stuart scientific SMP3 apparatus and are uncorrected.

\section{General procedure for the synthesis of $N^{\prime}$ - Benzylidene-2-(4-bromophenoxy)-2-methyl propane hydrazides (5a-5j)}

For the synthesis of azomathine derivatives, usinga modified literature method 2-(4Bromophenoxy)-2-methyl propanonic acid (1) and ethyl-2-(4-bromophenoxy)-2-methyl propanoate (2) were synthesized [11]. Ethyl-2-(4bromohenoxy)-2-methyl propanoate (2) was converted into 2-(4-bromophenoxy)-2-methyl hydrazide (3) by refluxing ethyl-2-(4bromophenoxy)-2-methyl propanoate (2) (0.01 $\mathrm{mol})$ with hydrazine hydrate $(0.01 \mathrm{~mol})$ in the presence of ethanol $(30 \mathrm{ml})$ for a period of $8 \mathrm{hrs}$. Completion of reaction was monitored by TLC (hexane: ethyl acetate). The contents of flask were reduced to half through distillation and cooled to room temperature. The remaining reaction mixture on constant stirring was acidified with $\mathrm{HCl}(5 \%)$ to get the product which was filtered, washed, dried and the yield of the product obtained was $80 \%$. Aromatic aldehydes or acetophenones ( $0.875 \mathrm{mmole})$, ethanol (30 $\mathrm{mL}$ ) and phosphoric acid (1-2 drops) with 2-(4bromophenoxy)-2-methyl hydrazide (3) (200 mg; $0.875 \mathrm{mmole}$ ) were refluxed in round bottom flask for the period of 3-4 hrs. When the reaction was completed (monitored by TLC), the content of flask was cooled to room temperature and evaporation of the solvent under vacuum yields the solid product. The residue was purified by recrystallization from ethyl alcohol.

\section{Biofilm inhibition assay}

The inhibition of biofilm formation was determined following a reported procedure [12]. The wells of a sterile 96-well flat bottomed tissue culture plates were filled with nutrient broth (100 $\mu \mathrm{L}$, Oxoid, UK), testing sample $(100 \mu \mathrm{L})$ and bacterial suspension $(20 \mu \mathrm{L})$ was inoculated. Negative control wells contained nutrient broth and microbial strain while positive control contained standard antibiotic Rifampicin and nutrient broth only. Tissue cultured Plates were incubated under aerobic condition for $24 \mathrm{~h}$ at $37^{\circ} \mathrm{C}$. Thereafter, each plate were treated with buffer of pH: 7.2 (phosphate buffer) for removal of non-adherent bacteria, for remaining bacteria all plates were treated with methanol. Afterward Crystal violet $(50 \%)$ was used for staining and excess stain rinsed off. The OD of each well was measured at $630 \mathrm{~nm}$ using micro plate reader (BioTek, USA) and bacterial growth inhibition (INH) percent was calculated as in Eq 1.

INH $(\%)=\left[100-\left(\mathrm{OD}_{630 \text { sample }} \times 100\right)\right] / \mathrm{OD}_{630 \text { control }} \cdot 1$

where $O D=$ optical density .

\section{Antioxidant assay}

The antioxidant activity of compounds was determined using 2, 2-diphenyl-1-picrylhydrazyl (DPPH) spectrophotometric method [13] with slight modification. $0.004 \%(1 \mathrm{~mL})$ of DPPH in methanol was added to sample solution $(3 \mathrm{~mL})$ and the mixture was kept in the dark for $30 \mathrm{~min}$. The decrease in absorbance was noted at 517 $\mathrm{nm}$. Ascorbic acid was taken as the standard, and inhibition (\%) was calculated as in Eq 2.

$D(\%)=\left(A_{0}-A_{1} \times 100\right) / A_{0} \ldots \ldots \ldots(2)$

where $A_{1}=$ absorbance of sample; $A_{0}=$ absorbance of blank.

\section{Mutagenic screening assay}

Mutagenicity of synthesized compounds was determined by Ames bacterial reverse-mutation method [14]. A fresh strain of Salmonella culture (test strain), sample solution, standard and reagent mixture were combined in the following ratio (Table 1).

Mutant strains of Salmonella (TA98 and TA100) were used. The prepared contents $(200 \mu \mathrm{L})$ was dispensed into each well of a 96-well micro titration plate and incubated at $37{ }^{\circ} \mathrm{C}$ for 4 days. 
Table 1: Ratio of standards and compounds

\begin{tabular}{lccccc}
\hline Variable & \multicolumn{5}{c}{ Volume used $(\mathbf{m L})$} \\
\cline { 2 - 6 } & Standard & Sample & Reagent mixture & Deionized water & Test strain \\
\hline Blank & - & - & 2.5 & 17.5 & - \\
Background & - & - & 2.5 & 17.5 & 0.005 \\
\hline Standard Mutagen & 0.1 & - & 2.5 & 17.4 & 0.005 \\
\hline (test) sample & - & 0.005 & 2.5 & 17.5 & 0.005 \\
\hline
\end{tabular}

The blank plate was first observed and the rest of plates were read only when all wells in the blank plate were colored purple-an indication that the assay was not contaminated. The background, standard, and test plates observed visually, all yellow, partial yellow or turbid wells were scored as positive wells while purple wells were scored as negative. For a compound to be mutagenic, the number of positive wells had to be more than twice the number of positive well in the background plate.

\section{RESULTS}

\section{Chemistry}

2-(4-Bromophenoxy)-2-methyl propane hydrazide (3), was synthesized by hydrazinolysis of ethyl 2-(4-bromophenoxy)-2-methyl propaneate (2) which was obtained by the esterification of 2(4-bromophenoxy)-2-methyl propanoic acid (1) in the presence of sulfuric acid as catalyst (Figure$1)$. Condensation of aromatic aldehydes and acetophenones with 2-(4-bromophenoxy)-2methyl propane hydrazide (3) in ethanol yields the benzylidene-2-(4-bromophenoxy)-2-methyl propane hydrazide (4a-4j) compounds, all the compounds were characterized by using different spectroscopic techniques (FTIR, ${ }^{1} \mathrm{H}$ NMR, ${ }^{13} \mathrm{C}$ NMR and mass spectrometry). Compounds (4a4j) are white to pale yellow crystalline solids. They exhibit sharp melting points and are fairly soluble in ethanol and other common organic solvents.

The presence of functional groups in compounds (4a-4j) were established using FTIR spectroscopy by comparing their spectra with precursors. Useful information obtained are as follows; the appearance of characteristic peak of $-\mathrm{NH}$ around $3240 \mathrm{~cm}^{-1}$ indicate the conversion of primary amine into secondary amine which is related to reported value of $3120-3330 \mathrm{~cm}^{-1}$ [15]. The absence of peak at range of 1700 $1750 \mathrm{~cm}^{-1}$ further confirmed the conversion of aldehyde $(-\mathrm{CHO})$ and acetophenone $(-\mathrm{C}=\mathrm{O})$ group into $-\mathrm{C}=\mathrm{N}$ - linkage [16]. Appearance of two other characteristic peaks between 1583 $1600 \mathrm{~cm}^{-1}$ and $1715-1759 \mathrm{~cm}^{-1}$ can be attributed to $\mathrm{C}=\mathrm{N}$ and $\mathrm{C}=\mathrm{O}$ respectively and are closely related with the literature value of acetate and azomethine moiety $\left(1590-1670 \mathrm{~cm}^{-1}\right.$ and $1720-1750 \mathrm{~cm}^{-1}$ ) [17]. The presence of these functional groups $\mathrm{NH}, \mathrm{C}=\mathrm{N}$ and $\mathrm{C}=\mathrm{O}$ agree with the proposed structures of desired derivatives.

${ }^{1} \mathrm{H}$ NMR spectra were recorded in DMSO-d6 and the values of chemical shifts are given in the experimental section. The important peaks of $\mathrm{NH}$ and methyl group $-\mathrm{C}\left(\mathrm{CH}_{3}\right)_{2}$ protons appears at 9.68 - $11.11 \mathrm{ppm}$ and $1.49-1.64 \mathrm{ppm}$ respectively. A strong singlet peak of $-\mathrm{CH}$ of the aldehyde group in $4 a, 4 b, 4 c, 4 d, 4 e$ and $4 f$ appears between 8.12 - 8.72 ppm [18]. Similarly, azomethine compounds synthesized from acetophenones $(\mathbf{4 g}, \mathbf{4 h}, \mathbf{4} \mathbf{i}$ and $\mathbf{4 j})$ also shows sharp singlet peak of $-\mathrm{CH}_{3}$ around $2.20-3.85$ ppm which are close to literature value of 2.50 ppm [19]. The azomethine ligands shows multiplet signals around $6.88-7.99 \mathrm{ppm}$ which can be assigned as protons of benzene ring. The presence of substituents on benzene ring greatly affects the chemical shift values as seen in $\mathbf{4 h}$ whereas the hydroxyl group present at the ortho position appears as a singlet at $9.64 \mathrm{ppm}$ which is close to the literature value of $10.22 \mathrm{ppm}$ [20]. In ${ }^{13} \mathrm{C}-\mathrm{NMR}$, the representative amide group peak in all compounds appears in the range of 168-155 ppm which matches reported value of 167 - 152 ppm. Similarly, aromatic carbon atoms were seen between 110-150 ppm depending on their electronic environment and non-aromatic carbon atoms found in the methoxy group of $\mathbf{4 b}$ and $4 \mathbf{i}$ were observed at 55.32ppm which correlates with reported value of $55.91 \mathrm{ppm}$ [21]. The methyl group in $\mathbf{4 g}, \mathbf{4 h}, \mathbf{4 i}$ and $\mathbf{4 j}$ shows a sharp signal at $25.29 \mathrm{ppm}$.

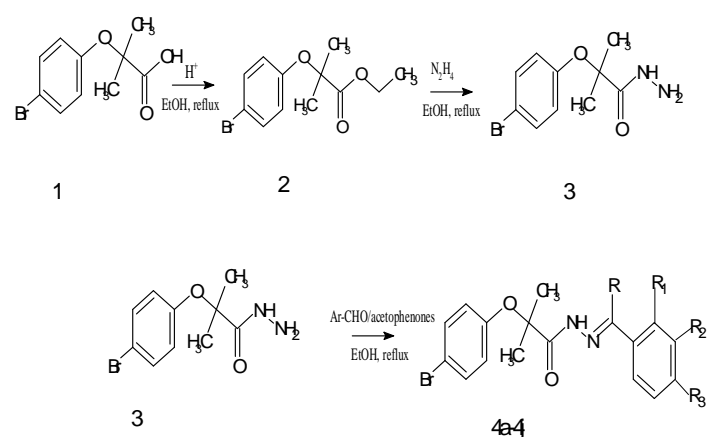

Figure 1: Synthesis of benzylidene-2-(4bromophenoxy)-2-methyl propane hydrazide 
4-Bromobenzylidene-2-(4-chlorophenoxy)-2methylpropanehydrazide (4a)

Light yellow crystals; Yield $72 \%$; m.p, 176$177^{\circ} \mathrm{C}$; IR $(\mathrm{KBr}) \mathrm{cm}^{-1}$ : 3241(NH), 1745(C=O), 1625(C=N); ${ }^{1} \mathrm{H}$ NMR (DMSO.d $\left.300 \mathrm{MHz}\right)$ : $9.74(\mathrm{~s}, 1 \mathrm{H}, \mathrm{NH}), 8.12(\mathrm{~s}, 1 \mathrm{H}, \mathrm{CH}), 7.76-7.68(\mathrm{~m}$, $2 \mathrm{H}, \mathrm{ArH}) 7.41-7.37(\mathrm{~m}, 2 \mathrm{H}, \mathrm{ArH}) 7.28-7.25(\mathrm{~m}$, $2 \mathrm{H}, \mathrm{ArH}), 6.95-6.91(\mathrm{~m}, 2 \mathrm{H}, \mathrm{ArH}), 1.62(\mathrm{~s}, 6 \mathrm{H}$, $\left.\mathrm{C}\left(\mathrm{CH}_{3}\right)_{2}\right) \cdot{ }^{13} \mathrm{C}$ NMR (DMSO, $\left.100 \mathrm{MHz}\right): 168.52$, $152.74,145.86,138.22,132.50,131.97,129.58$, 129.05, 128.73, 120.19, 82.33, 25.29; ESI可MS $\mathrm{m} / \mathrm{z}$ (\%): 396.01[M+1] ${ }^{+}$; Anal. Calcd for $\mathrm{C}_{17} \mathrm{H}_{16} \mathrm{BrClN}_{2} \mathrm{O}_{2}$ : C, 58.12; $\mathrm{H}, 4.59 ; \mathrm{N}, 7.97$; Found C, 58.09; H, 4.54; N, 8.06.

\section{2-(4-Bromophenoxy)-2-methoxybenzylidene-2- methylpropanehydrazide (4b)}

Off white powder; Yield:90 \%; m.p:188-189 $\mathrm{C}$ $(\mathrm{KBr}) \mathrm{cm}^{-1}$ : 3341(NH), 1758(C=O), 1583(C=N); ${ }^{1} \mathrm{H}$ NMR (DMSO. $\left.d_{6} 300 \mathrm{MHz}\right) \delta: 9.64(\mathrm{~s}, 1 \mathrm{H}, \mathrm{NH})$, $8.72(\mathrm{t}, J=0.9 \mathrm{HZ}, 1 \mathrm{H}, \mathrm{CH}), 7.91-7.68(\mathrm{~m}, 2 \mathrm{H}, \mathrm{Ar} H)$ 7.52-7.37(m, 2H, ArH) 7.28 -7.21 (m, 2H, ArH), 6.90-6.88 (m, 2H, ArH) 3.92(S, 3H, OCH $\left.H_{3}\right) 1.62$ (s, $\left.6 \mathrm{H}, \quad \mathrm{C}\left(\mathrm{CH}_{3}\right)_{2}\right) .{ }^{13} \mathrm{C}$ NMR (DMSO, $100 \mathrm{MHz}$ ): $168.52,158.35,152.74,145.88,132.50,129.58$, $128.93,126.04,120.19,113.75,82.33,55.32$, 25.29;ESIOMS $\mathrm{m} / \mathrm{z} \%): 392.1[\mathrm{M}+1]^{+}$;Anal. Calcd for: $\mathrm{C}_{18} \mathrm{H}_{19} \mathrm{BrN}_{2} \mathrm{O}_{3}$ : C, 62.32; $\mathrm{H}, 5.53 ; \mathrm{N}, 8.07$; Found $\mathrm{C}$ 62.42; H, 5.55; N, 7.97.

\section{2-(4-Bromophenoxy)-2-methyl-(4- nitrobenzylidene) propanehydrazide (4c)}

Light yellow crystal; Yield $80 \%$; m.p,158-160 ${ }^{\circ} \mathrm{C}$; IR $(\mathrm{KBr}) \quad \mathrm{cm}^{-1}: \quad 3424(\mathrm{NH}), \quad$ 1748(C=O), 1598(C=N); ${ }^{1} \mathrm{H}$ NMR (DMSO. $\left.d_{6} 300 \mathrm{MHz}\right) \delta$ : $9.95(\mathrm{~s}, 1 \mathrm{H}, \mathrm{NH}), 8.37(\mathrm{~s}, 1 \mathrm{H}, \mathrm{CH}), 8.26(\mathrm{~d}, J=$ $9.0 \mathrm{~Hz}, 2 \mathrm{H}, \operatorname{ArH}), 7.92(\mathrm{~d}, J=8.9 \mathrm{~Hz} 2 \mathrm{H}, \operatorname{ArH}) 7.30$ - $7.26(\mathrm{~m}, 2 \mathrm{H}, \operatorname{Ar} H), 6.94-6.92(\mathrm{~m}, 2 \mathrm{H}, \operatorname{Ar} H)$, $1.63\left(\mathrm{~s}, 6 \mathrm{H}, \mathrm{C}\left(\mathrm{CH}_{3}\right)_{2}\right)$; ${ }^{13} \mathrm{C}$ NMR (DMSO,100 $\mathrm{MHz}): 168.52,152.74,147.43,145.73,138.88$, $132.50,129.58,127.93,123.65,120.19,82.33$, 25.29; ESI回MS m/z (\%): 407.1[M+1]+; Anal. Calcd for: $\mathrm{C}_{17} \mathrm{H}_{16} \mathrm{BrN}_{3} \mathrm{O}_{4}$ : C, 56.44; $\mathrm{H}, 4.49 ; \mathrm{N}$, 11.61; Found C, 56.49; H, 4.55; N, 11.67.

\section{3-Chlorobenzylidene-2-(4-bromophenoxy)-2- methylpropanehydrazide (4d)}

Off white crystal; Yield $88 \%$; m.p, $152-154{ }^{\circ} \mathrm{C}$; IR $(\mathrm{KBr}) \quad \mathrm{cm}^{-1}: \quad 3236(\mathrm{NH}), \quad$ 1725(C=O), 1588(C=N); ${ }^{1} \mathrm{H} \quad$ NMR (DMSO. $\left.d_{6} 300 \mathrm{MHz}\right) \delta$ : $9.80(\mathrm{~s}, 1 \mathrm{H}, \mathrm{NH}), 8.18(\mathrm{~s}, 1 \mathrm{H}, \mathrm{CH}) 7.79(\mathrm{t}, J=0.9$ $\mathrm{Hz}, 1 \mathrm{H}, \operatorname{Ar} H), 7.61-7.58(\mathrm{~m}, 1 \mathrm{H}, \operatorname{Ar} H), 7.40-$ $7.25(\mathrm{~m}, 4 \mathrm{H}, \mathrm{Ar} H), 7.02-6.96(\mathrm{~m}, 2 \mathrm{H}, \operatorname{Ar} H)$, $1.59\left(\mathrm{~s}, 6 \mathrm{H}, \mathrm{C}\left(\mathrm{CH}_{3}\right)_{2}\right) \cdot{ }^{13} \mathrm{C}$ NMR (DMSO, 100 $\mathrm{MHz}$ ): 168.52, 152.74, 146.25, 134.28, 133.01, 132.50, 131.26, 129.58, 128.28, 126.49, 124.18,
120.19, 82.33, 25.29; ESI0MS $\mathrm{m} / \mathrm{z}$ (\%):395.6[M+1] $;$; Anal. Calcd for: $\mathrm{C}_{17} \mathrm{H}_{16} \mathrm{BrClN}_{2} \mathrm{O}_{2}: \mathrm{C}, 58.13 ; \mathrm{H}, 4.59 ; \mathrm{N}, 7.98$;

Found C, 58.12; H, 4.56; N, 8.01.

\section{2-(4-Bromophenoxy)-2-methyl-(3-}

nitrobenzylidene) propanehydrazide (4e)

Pale yellow powder; Yield 87\%; m.p:175-177 C;IR $(\mathrm{KBr}) \mathrm{cm}^{-1}: 3041(\mathrm{NH}), \quad 1723(\mathrm{C}=\mathrm{O}), \quad 1600(\mathrm{C}=\mathrm{N})$; ${ }^{1}$ HNMR (DMSO. d $\left.300 \mathrm{MHz}\right) \delta: 10.11(\mathrm{~s}, 1 \mathrm{H}, \mathrm{NH})$, $8.46(\mathrm{td}, J=1.8,1.1 \mathrm{~Hz}, 1 \mathrm{H}, \mathrm{CH}), 8.12-8.01(\mathrm{~m}$, $1 \mathrm{H}, \operatorname{ArH}), 8.01-7.90(\mathrm{~m}, 2 \mathrm{H}, \operatorname{Ar} H), 7.78$ (t, $J=7.8$ $\mathrm{Hz}, 1 \mathrm{H}, \operatorname{Ar} H), 7.40-7.29(\mathrm{~m}, 2 \mathrm{H}, \mathrm{ArH}), 6.85-6.74$ $(\mathrm{m}, 2 \mathrm{H}, \mathrm{ArH}), 1.52\left(\mathrm{~s}, 6 \mathrm{H}, \mathrm{C}\left(\mathrm{CH}_{3}\right)_{2}\right) \cdot{ }^{13} \mathrm{C} \mathrm{NMR}$ (DMSO, $100 \mathrm{MHz}$ ): 168.52, 152.74, 147.43, 145.73, $138.88,132.50,129.58,127.93,123.65,120.19$, 82.33, 25.29;ESIIIMS $\mathrm{m} / \mathrm{z} \quad \%$ ):407.0[M+1] ;Anal.Calcd for: $\mathrm{C}_{17} \mathrm{H}_{16} \mathrm{BrN}_{3} \mathrm{O}_{4}$ : C, 56.44; H, 4.46; N, 11.61; Found C, 56.52; H, 4.55; N, 11.54 .

4-Bromobenzylidene-2-(4-bromophenoxy)-2methylpropanehydrazide (4f)

White crystal; Yield:69 \%; m.p: $201-203^{\circ} \mathrm{C}$;IR $(\mathrm{KBr}) \mathrm{cm}^{-1}: 3041(\mathrm{NH}), 1721(\mathrm{C}=\mathrm{O}), 1583(\mathrm{C}=\mathrm{N})$; ${ }^{1}$ HNMR (DMSO. $\left.d_{6} 300 \mathrm{MHz}\right) \delta: 11.66(\mathrm{~s}, 1 \mathrm{H}$, $\mathrm{NH}), 8.37(\mathrm{~s}, 1 \mathrm{H}, \mathrm{CH}), 7.85-7.58(\mathrm{~m}, 2 \mathrm{H}, \mathrm{ArH})$, $7.75-7.68(\mathrm{~m}, 2 \mathrm{H}, \mathrm{Ar} H), 7.40-7.29(\mathrm{~m}, 2 \mathrm{H}$, $\mathrm{ArH}), 6.82-6.72(\mathrm{~m}, 2 \mathrm{H}, \operatorname{Ar} H), 1.53(\mathrm{~s}, 6 \mathrm{H}, \mathrm{C}$ $\left.\left(\mathrm{CH}_{3}\right)_{2}\right) \cdot{ }^{13} \mathrm{C}$ NMR (DMSO,100 MHz): 168.52, $152.74,145.86,138.22,132.50,131.97,129.58$, 129.05, 128.73, 120.19, 82.33, 25.29; ESI回MS $\mathrm{m} / \mathrm{z}$ (\%):441.9[M+1]+; Anal. Calcd for: $\mathrm{C}_{17}$ $\mathrm{H}_{16} \mathrm{Br}_{2} \mathrm{~N}_{2} \mathrm{O}_{2}: \mathrm{C}, 51.60 ; \mathrm{H}, 4.09 ; \mathrm{N}, 7.07$; Found $\mathrm{C}$, 51.68; H, 4.15; N, 6.97.

\section{2-(4-Bromophenoxy)-(1-(4-chlorophenyl) ethylidene)-2-methylpropanehydrazide (4g)}

Off white powder; Yield: $79 \%$; m.p: 212-213 ${ }^{\circ} \mathrm{C}$; IR (KBr) cm $\mathrm{cm}^{-1}: \quad 3214(\mathrm{NH}), \quad$ 1757(C=O), 1592(C=N); ${ }^{1} \mathrm{HNMR}$ (DMSO.d $\left.300 \mathrm{MHz}\right) \delta$ : $10.01(\mathrm{~s}, 1 \mathrm{H}, \mathrm{NH}), 7.66-7.56(\mathrm{~m}, 2 \mathrm{H}, \mathrm{ArH})$, 7.33 (ddt, $J=10.3,8.9,1.7 \mathrm{~Hz}, 4 \mathrm{H}, \operatorname{ArH}), 7.00-$ $6.91(\mathrm{~m}, 2 \mathrm{H}, \mathrm{ArH}), 2.27\left(\mathrm{~s}, 3 \mathrm{H}, \mathrm{CH}_{3}\right), 1.51(\mathrm{~s}, 6 \mathrm{H}$, $\mathrm{C}\left(\mathrm{CH}_{3}\right)_{2 .}$.) ${ }^{13} \mathrm{C}$ NMR (DMSO100 MHz): 168.52, 152.74, 150.40, 134.75, 134.26, 132.50, 129.74, $129.58,129.22,128.21,127.43,120.19,82.33$, 25.29; ESI Calcd for: $\mathrm{C}_{18} \mathrm{H}_{18} \mathrm{BrClN}_{2} \mathrm{O}_{2}$ : C, 59.18; $\mathrm{H}, 4.97 ; \mathrm{N}$, 7.67; Found C, 59.22; H, 4.02; N, 7.66.

\section{2-(4-Bromophenoxy)-(1-(2-hydroxyphenyl) ethylidene)-2-methylpropanehydrazide $(4 \mathrm{~h})$}

White powder; Yield: $77 \%$; m.p:188-190 ${ }^{\circ} \mathrm{C}$; IR $(\mathrm{KBr}) \mathrm{cm}^{-1}$ : 3233(NH), 1759(C=O), 1598(C=N); ${ }^{1} \mathrm{H}$ NMR (DMSO. $\left.d_{6} 300 \mathrm{MHz}\right) \delta: 12.63(\mathrm{~s}, 1 \mathrm{H}$, $\mathrm{NH}), 9.64(\mathrm{~s}, 1 \mathrm{H}, \mathrm{OH}), 7.47-7.44(\mathrm{dd}, J=7.7$, 
$1.6 \mathrm{~Hz}, 1 \mathrm{H}, \mathrm{ArH}), 7.34-7.28(\mathrm{~m}, 3 \mathrm{H}, \mathrm{ArH}), 7.06$ - $7.03(\mathrm{~m}, 1 \mathrm{H}, \mathrm{ArH}), 6.98-6.86(\mathrm{~m}, 3 \mathrm{H}, \mathrm{ArH}) 2.30$ (s, 3H, $\left.\mathrm{CH}_{3}\right), 1.62\left(\mathrm{~s}, 6 \mathrm{H}, \mathrm{C}\left(\mathrm{CH}_{3}\right)_{2}\right) .{ }^{13} \mathrm{C} \mathrm{NMR}$ (DMSO,100 MHz): 169.14, 156.83, 156.26, $152.74,132.50,131.90,129.58,128.88,120.27$, 120.19, 119.01, 115.15, 82.42, 25.29, 14.41;ESI?MS m/z (\%):392.1[M+1] $]^{+}$; Anal. Calcd for: $\mathrm{C}_{18} \mathrm{H}_{19} \mathrm{BrN}_{2} \mathrm{O}_{3}$ : C, 62.33; $\mathrm{H}, 5.52 ; \mathrm{N}, 8.07$; Found $\mathrm{C}, 62.25 ; \mathrm{H}, 5.44 ; \mathrm{N}, 8.19$.

\section{2-(4-Bromophenoxy)-(1-(4-methoxyphenyl) ethylidene)-2-methylpropanehydrazide (4i)}

Pale yellow powder; Yield: 62\%; m.p:191-192 C; IR $(\mathrm{KBr}) \mathrm{cm}^{-1}: \quad 3224(\mathrm{NH}), \quad 1715(\mathrm{C}=\mathrm{O})$, 1598(C=N); ${ }^{1} \mathrm{HNMR}$ (DMSO. $\left.\mathrm{d}_{6} 300 \mathrm{MHz}\right) \delta: 9.56$ (s, $1 \mathrm{H}, \mathrm{NH}), 7.85-7.81(\mathrm{~m}, 2 \mathrm{H}, \mathrm{ArH}), 7.31-$ $7.26(\mathrm{~m}, 2 \mathrm{H}, \mathrm{ArH}), 6.98-6.90(\mathrm{~m}, 4 \mathrm{H}, \mathrm{ArH}), 3.85$ (s, 3H, $\left.\mathrm{CH}_{3}\right), 3.20$ (s, 3H,OCH$), 1.62(\mathrm{~s}, 6 \mathrm{H}$, $\left.\mathrm{C}\left(\mathrm{CH}_{3}\right)_{2}\right) \cdot{ }^{13} \mathrm{C}$ NMR (DMSO,100 MHz): 169.14, 161.36, 152.74, 148.77, 132.50, 129.58, 129.47, 128.98, 120.19, 112.93, 82.42, 55.32, 25.29, 13.89;ESI?MS $\mathrm{m} / \mathrm{z}(\%): 406.1[\mathrm{M}+1]+;$ Anal. Calcd for: $\mathrm{C}_{19} \mathrm{H}_{21} \mathrm{BrN}_{2} \mathrm{O}_{3}: \mathrm{C}, 58.12 ; \mathrm{H}, 4.59 ; \mathrm{N}, 7.97$; Found C, 58.22; H, 4.69; N, 8.07.

\section{2-(4-Bromophenoxy)-2-methyl-(1-phenylethyl- idene) propanehydrazide (4j)}

White crystalline powder; Yield: 81\%; m.p:202$204^{\circ} \mathrm{C}$; IR (KBr) cm ${ }^{-1}$ : 3321(NH), 1759(C=O), 1591 $(\mathrm{C}=\mathrm{N})$; ${ }^{1} \mathrm{HNMR}$ (DMSO. $\left.\mathrm{d}_{6} 300 \mathrm{MHz}\right) \mathrm{d}$ : $9.63(\mathrm{~s}, 1 \mathrm{H}, \mathrm{NH}), 7.87-7.83(\mathrm{~m}, 2 \mathrm{H}, \mathrm{ArH}), 7.42-$ $7.39(\mathrm{~m}, 3 \mathrm{H}, \mathrm{ArH}), 7.31-7.26(\mathrm{~m}, 2 \mathrm{H}, \mathrm{ArH}) 6.98-$ $6.93(\mathrm{~m}, 2 \mathrm{H}, \mathrm{ArH}), 2.23\left(\mathrm{~s}, 3 \mathrm{H}, \mathrm{CH}_{3}\right), 1.62(\mathrm{~s}, 6 \mathrm{H}$, $\left.\mathrm{C}\left(\mathrm{CH}_{3}\right)_{2}\right) \cdot{ }^{13} \mathrm{C}$ NMR (DMSO, $\left.100 \mathrm{MHz}\right): 169.14$, 152.74, 148.92, 136.63, 132.50, 129.90, 129.58, 127.51, 127.28, 120.19, 82.42, 25.29, 13.89; ESI?MS $m / z): 375.1[\mathrm{M}+1]^{+}$; Anal. Calcd for: $\mathrm{C}_{18} \mathrm{H}_{19} \mathrm{BrN}_{2} \mathrm{O}_{2}$ : C, 63.24; $\mathrm{H}, 5.89 ; \mathrm{N}, 7.76$; Found C, 63.12; H, 5.75; N, 7.88.

All the synthesized compounds were subjected to biofilm inhibition, antioxidant and mutagenic assay using well-defined literature methods.

\section{Biofilm inhibition activity}

A microtitre-plate method was used to determine the inhibitory activity of compounds in biofilm formation. All the compounds showed sensitivity against gram-positive and gram-negative strains of bacteria. Rifimpacin (Oxoid, UK) were used as standard antibiotic (positive control) to compare sensitivity of azomethin compounds in biofilm inhibition. It was found that the inhibition assay depends upon the ability of azomethine compounds to form a chelate with bacterial strains and inhibit their growth [22]. Table 2 shows that all compounds are more active against $E$. coli gram-negative bacterial strain than the gram-positive bacterial strain.

Table 2: Biofilm inhibition percent of benzylidene-2-(4bromophenoxy)-2-methyl propane hydrazides

\begin{tabular}{lcc} 
Compound & $\begin{array}{c}\text { Biofilm } \\
\text { inhibition \% } \\
\text { (S.aureus) } \\
\text { Gram +ive } \\
\text { strain }\end{array}$ & $\begin{array}{c}\text { Biofilm inhibition } \\
\text { \% (E.coli) } \\
\text { Gram -ive sttrain }\end{array}$ \\
\hline $\mathbf{4 a}$ & 57.84 & 75.33 \\
$\mathbf{4 b}$ & 77.28 & 79.22 \\
$\mathbf{4 c}$ & 57.39 & 75.92 \\
$\mathbf{4 d}$ & 57.29 & 49.46 \\
$\mathbf{4 e}$ & 55.65 & 74.14 \\
$\mathbf{4 f}$ & 43.18 & 75.63 \\
$\mathbf{4 g}$ & 63.31 & 57.07 \\
$\mathbf{4 h}$ & 57.01 & 60.41 \\
$\mathbf{4 i}$ & 75.51 & 77.05 \\
$\mathbf{4 j}$ & 61.26 & 65.22 \\
Positive & $\mathbf{8 4 . 5 0}$ & $\mathbf{8 9 . 0 1}$ \\
control & & \\
(Rifimpacin) & & \\
\hline
\end{tabular}

\section{Anti-oxidant activity}

The anti-oxidant activity was evaluated by the radical scavenging ability of compounds by using 1, 1-diphenyl-2-picrylhydrazyl (DPPH). In this series, the scavenging potential lies between moderate to good yield as shown in Table 3 when compare with the scavenging potential of standard compound. In Compound $\mathbf{4 b}$ and $\mathbf{4} \mathbf{i}$ the presence of methoxy group at the ortho and para position of the aromatic ring enhance the scavenging ability.

Table 3: Anti-oxidant activity of benzylidene-2-(4bromophenoxy)-2-methyl propane hydrazide

\begin{tabular}{lc} 
Compound & DPPH (\%) \\
\hline $\mathbf{4 a}$ & 51.22 \\
$\mathbf{4 b}$ & 89.45 \\
$\mathbf{4 c}$ & 23.96 \\
$\mathbf{4 d}$ & 56.56 \\
$\mathbf{4 e}$ & 55.28 \\
$\mathbf{4 f}$ & 62.80 \\
$\mathbf{4 g}$ & 51.00 \\
$\mathbf{4 h}$ & 71.77 \\
$\mathbf{4 i}$ & 82.54 \\
$\mathbf{4 j}$ & 67.12 \\
Ascorbic acid & $\mathbf{9 2 . 4 7}$
\end{tabular}

\section{Mutagenic activity}

Mutagenic screening of compounds was evaluated by Ames bacterial reverse-mutation method. All the compounds were sensitive against both strains of salmonella TA98 and TA 100. The result (Table.4) shows that most of the compounds were non-mutagenic or mutagenic for only one strain under assay condition. The 
Table 4: Mutagenic screening of benzylidene-2-(4-bromophenoxy)-2-methyl propane hydrazide

\begin{tabular}{|c|c|c|c|c|}
\hline \multirow[t]{2}{*}{ Variable } & \multirow{2}{*}{$\begin{array}{c}\text { TA } 98 \\
\text { No. of + ve } \\
\text { wells/total } \\
\text { no. of wells }\end{array}$} & \multicolumn{3}{|c|}{ TA100 } \\
\hline & & Result & $\begin{array}{c}\text { No. of +ve } \\
\text { wells/total no. } \\
\text { of wells }\end{array}$ & Result \\
\hline Standard & $84 / 96$ & Mutagenic & $79 / 96$ & Mutagenic \\
\hline Background & $11 / 96$ & Non-mutagenic & $10 / 96$ & Non-mutagenic \\
\hline $4 a$ & $10 / 96$ & Non-mutagenic & $26 / 96$ & Mutagenic \\
\hline $4 b$ & 9/96 & Non-mutagenic & $10 / 96$ & Non-mutagenic \\
\hline $4 c$ & $10 / 96$ & Non-mutagenic & $16 / 96$ & Non-mutagenic \\
\hline $4 d$ & $22 / 96$ & Mutagenic & $24 / 96$ & Mutagenic \\
\hline $4 e$ & $22 / 96$ & Mutagenic & $10 / 96$ & Non-mutagenic \\
\hline $4 f$ & $8 / 96$ & Non-mutagenic & $8 / 96$ & Non-mutagenic \\
\hline $4 g$ & $5 / 96$ & Non-mutagenic & $20 / 96$ & Mutagenic \\
\hline $4 h$ & $10 / 96$ & Non-mutagenic & $8 / 96$ & Non-mutagenic \\
\hline $4 i$ & $11 / 96$ & Non-mutagenic & $9 / 96$ & Non-mutagenic \\
\hline $4 j$ & $7 / 96$ & Non-mutagenic & $10 / 96$ & Non-mutagenic \\
\hline
\end{tabular}

compound $\mathbf{4 d}$ only shows greater interaction for DNA to make it stable and causes genetic mutation [23].

\section{DISCUSSION}

It was found that organic ligands having azomethine linkage were synthesized by the condensation reaction of amines (primary or secondary) with benzaldehyde/acetophenones. They are diverse class of compounds which have broad range of biological activities such as antibacterial, antifungal, enzyme inhibition, antitumor and antipyretic activities [24]. All the compounds were characterized by spectroscopic techniques. For functional group confirmation, in FTIR spectra the characteristic peak of $-\mathrm{NH}$ around $3240 \mathrm{~cm}^{-1}$ and azomethine appears at range of $1530-1600 \mathrm{~cm}^{-1}$ confirmed the synthesis of product, similarly in ${ }^{1} \mathrm{H}$ NMR the peak of $\mathrm{NH}_{2}$ around $4.50-5.20$ ppm was absent, the two prominent singlet peaks of $-\mathrm{CH}$ at range of $8.12-8.72 \mathrm{ppm}$ and six protons of $-\left(\mathrm{CH}_{3}\right)_{2}$ support the presence of fibrate moiety and azomethine linkage in benzylidine compound.

The results of biofilm inhibition reveled that all the ligands were active against gram-negative bacterial strain as compare to gram-positive strain. Literature shows that bacterial cell wall is composed of peptidoglycan which is thicker in Gram-positive strains and thinner in Gramnegative strains [25] due to which ligands can easily penetrate through the cell wall of gramnegative bacteria as compare to gram-positive bacteria and disturb cell growth to biofilm formation. Similarly ligands face a barrier for diffusion in Gram positive strain of bacteria [26] and was responsible for the low activity shown by some of the compounds. The compound $\mathbf{4 b}$ and 4i behave more potent as compare to all other compounds, it may be due to the presence of oxidative methoxy group whose strong oxidative nature crucially attacked the cell wall of microbes and damaged its growth. [27]

The azomethine compounds have an efficient conjugated system of delocalized electrons and form a stable radicals [28] which makes them an excellent antioxidant. The results in Table 3 shows that the compounds substituted with electron donating group as in $4 b$ and $4 i$ have more scavenging activity, while the presence of electron withdrawing substituents $(\mathrm{Cl}, \mathrm{Br})$ have the least effect on delocalization and hence, they shows poor activity.

In mutagenic screening result, it was found that most of the compounds shows different mutagenicity behavior against both strains of Salmonella TA98 and TA 100.

Literature shows that the position and nature of substituents on phenyl ring greatly effect the mutagenic results [29] as in compound $4 \mathrm{~d}$ chloro group is present at Meta position of phenyl ring and causes mutagenicity against both strains of Salmonella TA98 and TA 100 while in compound $4 \mathrm{e}$ nitro group is also present at meta position but it is mutagenic only for one strain. It shows that chloro and nitro groups at meta position creates greater interaction with DNA and becomes stable [30] and this stability causes genetic mutation in bacterial strains.

\section{CONCLUSION}

Azomethine derivatives of benzylidene-2-(4bromophenoxy)-2-methyl propane hydrazide have been successfully synthesized by following well-defined procedures and their structures confirmed by IR, NMR and mass spectrometry. 
These compounds exhibit significant biological activities which are linked to the position and type of substituents on the structure of azomethine compounds. These findings may be helpful in the synthesis of more potent bioactive azometine compounds.

\section{DECLARATIONS}

\section{Acknowledgement}

The authors acknowledge Higher Education Commission of Pakistan for financial grant to Quaid Azam University Islamabad for spectral analysis and University of Agriculture Faisalabad for providing facilities in biological screening.

\section{Conflict of interest}

No conflict of interest is associated with this work.

\section{Contribution of authors}

We declare that this work was done by the authors named in this article and all liabilities pertaining to claims relating to the content of this article will be borne by the authors.

\section{Open Access}

This is an Open Access article that uses a funding model which does not charge readers or their institutions for access and distributed under the terms of the Creative Commons Attribution License (http://creativecommons.org/licenses/by/ 4.0) and the Budapest Open Access Initiative (http://www.budapestopenaccessinitiative.org/rea d), which permit unrestricted use, distribution, and reproduction in any medium, provided the original work is properly credited.

\section{REFERENCES}

1. Li JJ. Bargellini reaction. Name Reactions: A Collection of Detailed Reaction Mechanisms. Springer, Berlin, Heidelberg 2006; pp 24-25.

2. Yamazaki $Y$, Abe K, Toma $T$, Nishikawa M, Ozawa $H$, Okuda A, Araki T, Oda S, Inoue K, Shibuya K, Staels B. Design and synthesis of highly potent and selective human peroxisome proliferator-activated receptor a agonists. Bioorg Med Chem Lett 2007; 17(16): 46894693.

3. Desai RC, Metzger E, Santini C, Meinke PT, Heck JV, Berger JP, MacNaul KL, Cai TQ, Wright SD, Agrawal A, Moller DE. Design and synthesis of potent and subtypeselective PPARa agonists. Bioorg Med Chem Lett 2006; 16(6): 1673-1678.
4. Cvetovich RJ, Chung JY, Kress MH, Amato JS, Matty L, Weingarten MD, Tsay FR, Li Z, Zhou G. An efficient synthesis of a dual PPAR $\alpha / \gamma$ agonist and the formation of a sterically congested $\alpha$-aryloxyisobutyric acid via a Bargellini reaction. J Org Chem 2005; 70(21):85608563.

5. Dolaz M, McKee V, Golcu A, Tumer M. Synthesis, Structural Characterization, Spectroscopic and Electrochemical Studies of $N, \quad N^{\prime}-b i s \quad[(2,4-$ dimethoxyphenyl) methylidene] butane-1, 4-diamine. Curr Org Chem 2010; 14(3): 281-288.

6. Pingali $H$, Jain $M$, Shah $S$, Makadia $P$, Zaware $P$, Goel $A$, Patel M, Giri S, Patel H, Patel P. Design and synthesis of novel oxazole containing 1, 3-Dioxane-2-carboxylic acid derivatives as PPAR $\alpha / y$ dual agonists. Bioorg Med Chem 2008; 16(15): 7117-7127.

7. Liu W, Liu K, Wood HB, McCann ME, Doebber TW, Chang $\mathrm{CH}$, Akiyama TE, Einstein M, Berger JP, Meinke $P T$. Discovery of a peroxisome proliferator activated receptor Y (PPARY) modulator with balanced PPARa activity for the treatment of type 2 diabetes and dyslipidemia. J Med Chem 2009; 52(14): 4443-4453.

8. Sashidhara KV, Palnati GR, Dodda RP, Sonkar $R$, Khanna AK, Bhatia G. Discovery of amide based fibrates as possible antidyslipidemic and antioxidant agents. Eur J Med Chem 2012; 57: 302-310.

9. Navarrete-Vázquez G, Alaniz-Palacios A, HidalgoFigueroa S, González-Acevedo C, Ávila-Villarreal G, Estrada-Soto S, Webster SP, Medina-Franco JL, LópezVallejo F, Guerrero-Álvarez J, Tlahuext H. Discovery, synthesis and in combo studies of a tetrazole analogue of clofibric acid as a potent hypoglycemic agent. Bioorg Med Chem Lett 2013; 23(11): 3244-3247.

10. Akhter M, Husain A, Azad B, Ajmal M. Aroylpropionic acid based 2, 5-disubstituted-1, 3, 4-oxadiazoles: Synthesis and their anti-inflammatory and analgesic activities. Eur J Med Chem 2009; 44(6): 2372-23728.

11. Rehman MZ, Kazi AA, Siddiqui HL, Kashmiri MA, Ahmad VU. An efficient method for the synthesis of alkyl 2-(4benzoylphenoxy)-2-methyl propanoates. J Chem Soc Pak 2007; 29(4): 352-356.

12. Shahid S A, Anwar F, Shahid M, Majeed N, Azam A, Bashir M,Shakir I. Laser-Assisted synthesis of Mn 0.50 $\mathrm{Zn} 0.50 \mathrm{Fe} 204$ nanomaterial: characterization and in vitro inhibition activity towards bacillus subtilis biofilm. $J$ Nanomater 2015; 16(1): 111-116.

13. Bellik Y. Total antioxidant activity and antimicrobial potency of the essential oil and oleoresin of Zingiber officinale Roscoe. Asian Paci J Tropi Dis 2014; 4(1): 4044.

14. Tejs S. The Ames test: a methodological short review. Environ Biotech 2008; 4: 7-14.

15. Bharti SK, Nath G, Tilak R, Singh SK. Synthesis, antibacterial and anti-fungal activities of some novel Schiff bases containing 2, 4-disubstituted thiazole ring. Eur J Med Chem 2010; 45(2): 651-660

16. Alaghaz AN, Bayoumi HA, Ammar YA, Aldhlmani SA. Synthesis, characterization, and antipathogenic studies

Trop J Pharm Res, June 2019; 18(6): 1233 
of some transition metal complexes with $\mathrm{N}$, O-chelating Schiff's base ligand incorporating azo and sulfonamide Moieties. J Mol Stru 2013; 1035: 383-399.

17. Shanty AA, Philip JE, Sneha EJ, Kurup MR, Balachandran S, Mohanan PV. Synthesis, characterization and biological studies of Schiff bases derived from heterocyclic moiety. Bioorg chem 2017; 70: 67-73.

18. Shelke VA, Jadhav SM, Patharkar VR, Shankarwar SG, Munde AS, Chondhekar TK. Synthesis, spectroscopic characterization and thermal studies of some rare earth metal complexes of unsymmetrical tetradentate Schiff base ligand. Arabian J Chem 2012; 5(4): 501-507.

19. Kotkar SN, Juneja HD. Synthesis, Characterization, and Antimicrobial Studies of N, O Donor Schiff Base Polymeric Complexes. J Chem 2013; 2013:

20. Chohan ZH, Sumrra SH, Youssoufi MH, Hadda TB. Metal based biologically active compounds: Design, synthesis, and antibacterial/antifungal/cytotoxic properties of triazole-derived Schiff bases and their oxovanadium (IV) complexes. Eur J Med Chem 2010; 45(7): 2739-2747.

21. Trávníček Z, Buchtík R, Nemec I. Novel Schiff Bases Based on the Quinolinone Skeleton: Syntheses, X-ray Structures and Fluorescent Properties. Molecules 2014; 19(9):13509-13525.

22. Evans $M$, Rees $A$. The myotoxicity of statins. Curr Opin Lipid 2002; 13(4): 415-420.
23. Özkay Y, Incesu Z, Işıkdağ $i$, Yeşilkaya $M$. Antiproliferative effects of some $\mathrm{N}$-benzylideneanilines. Cell Biochem. Funct. 2008; 26(1): 102-106.

24. Tobriya, S K. Biological Applications of Schiff base and its Metal Complexes-A Review. Int J Sci Res 2014; 3(9): 1254-1256.

25. Sangshetti JN, Khan FA, Patil RH, Marathe SD, Gade $W N$, Shinde DB. Biofilm inhibition of linezolid-like Schiff bases: synthesis, biological activity, molecular docking and in silico ADME prediction. Bioorg Med Chem Lett 2015; 25(4): 874-880.

26. Ejiah FN, Fasina TM, Familoni OB, Ogunsola FT. Substituent effect on spectral and antimicrobial activity of Schiff bases derived from amino benzoic acids. Adv Bio Chem 2013; 3(05): 475-479.

27. Nel AE, Mädler L, Velegol D, Xia T, Hoek EM, Somasundaran P, Klaessig F, Castranova V, Thompson $M$. Understanding biophysicochemical interactions at the nano-bio interface. Nat Mater 2009; 8(7): 543-557.

28. Iftikhar B, Javed K, Khan MS, Akhter Z, Mirza B, Mckee $V$. Synthesis, characterization and biological assay of Salicylaldehyde Schiff base Cu (II) complexes and their precursors. J Mol Struct 2018; 1155: 337-348.

29. Aydoğan, G, Kutlu, M. Mutagenic activities of ten imidazole derivatives in Salmonella typhimurium. Biologia 2007; 62(1): 6-12.

30. Alanyali FS, Artagan Ö, Yuksel S. Mutagenicity Studies of some Substituted Benzylideneaniline Derivatives. Int J Pharmac 2011; 7(2): 278-282. 